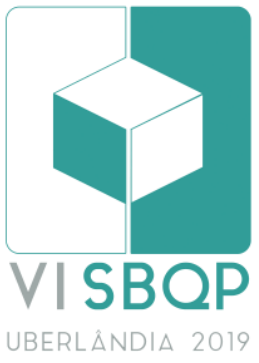

\title{
BIM (8D) COMO FERRAMENTA DE GESTÃO EM SEGURANÇA OCUPACIONAL: PERSPECTIVAS DE USO
}

\author{
SILVA, Thiago \\ Universidade de Pernambuco, e-mail: thsilva05@gmail.com \\ MANTA, Rafael \\ Universidade de Pernambuco, e-mail: rafaelmlsht@poli.br \\ TETI, Bruno \\ Universidade de Pernambuco, e-mail: bruno_teti@hotmail.com \\ MELHADO, Silvio Burratino \\ Universidade de São Paulo, e-mail: silvio_melhado@poli.usp.br \\ BARKOKÉBAS JUNIOR, Béda \\ Universidade de Pernambuco, e-mail: beda.jr@upe.br \\ LAFAYETTE, Kalinny \\ Universidade de Pernambuco, e-mail: klafayette@poli.br
}

\begin{abstract}
RESUMO
No Brasil, apenas a indústria da Construção Civil, registrou em 2017 um total de 30.025 acidentes. Parte desses acidentes poderiam ser evitadas através da prevenção através do projeto. O BIM vai muito além da criação de modelos 3D, com informações incorporadas atuando como uma importante ferramenta no auxilio de tomada de decisões e prevenção de acidentes através do projeto. O objetivo deste artigo é avaliar a percepção e utilização do BIM como ferramenta de gestão em Segurança do Trabalho pelos profissionais de Segurança. Foram elaboradas duas hipóteses: uma acerca da percepção dos benefícios do BIM sobre as razões de sua implantação na gestão da Segurança e outra em função das barreiras de implantação. Para investigar e tentar provar as hipóteses, um questionário foi aplicado aos profissionais de Segurança do Trabalho e um modelo de interação com multivariáveis foi desenvolvido seguindo os critérios do método Partial Least Square. O perfil qualitativo dos respondentes apresentou resultados no geral negativos acerca do conhecimento do BIM 8D e futuras perspectivas para $O$ mercado, sempre acima de $70 \%$ dos participantes. A amostragem possui $90 \%$ de confiança e margem de erro de $10,91 \%$ e o modelo apresentou validade, mas não se confirma em função de $H 2$, que obteve variância média extraída de 0,265 . O valor de $R^{2}$ para o constructo benefícios foi de $61,7 \%$, suficiente para estudos dessa natureza, qualificando os resultados do modelo como significativo sobre as "Razões de Implantação do BIM na gestão da SST".
\end{abstract}

Palavras-chave: Building Information Model, Segurança no trabalho, BIM 8D, Prevenção de acidentes, Projeto.

\begin{abstract}
In Brazil, only the Civil Construction industry registered in 2017 a total of 30,025 accidents. Part of these accidents could be avoided by prevention through the project. BIM goes far beyond the creation of 3D models, with embedded information acting as an important tool in aiding decision making and accident prevention through design. The aim of this paper is to evaluate the perception and use of BIM as a management tool in Occupational Safety by Safety professionals. Two hypotheses were elaborated: one about the perception of the benefits of BIM, over the reasons for its implementation in Safety and Security, and another about the perception of BIM barriers over the reasons for its implementation in safety. To investigate and try to prove the
\end{abstract}

SILVA, T.; MANTA, R.; TETI, B.; MELHADO, S.; BARKOKÉBAS JUNIOR, B.; LAFAYETTE, K. BIM (8D) como ferramenta de gestão em segurança ocupacional: perspectivas de uso. In: SIMPÓSIO BRASILEIRO DE QUALIDADE DO PROJETO NO AMBIENTE CONSTRUÍDO, 6., 2019, Uberlândia. Anais... Uberlândia: PPGAU/FAUeD/UFU, 2019. p 636-646. DOI https://doi.org/10.14393/sbqp19059. 
hypotheses, a questionnaire was applied to the Occupational Safety professionals and a multivariate interaction model was developed following the criteria of the Partial Least Square method. The qualitative profile of the respondents presented generally negative results about the knowledge of BIM 8D and future perspectives for the market, always above $70 \%$ of the participants. The sampling has a $90 \%$ confidence and a margin of error of $10.91 \%$ and the model has validity, but it is not confirmed as a function of $\mathrm{H} 2$, which obtained an average variance of 0.265. The $R^{2}$ value for the construct "Benefits" was $61.7 \%$, sufficient for studies of this nature, qualifying the results of the model as significant on the "Reasons for BIM implementation in OSH management".

Keywords: Building Information Model, safety at work, accident prevention, BIM 8D, design.

\section{INTRODUÇÃO}

De acordo com dados recentes da Organização Internacional do Trabalho OIT (ILO, 2018), 2,78 milhões de mortes ocorrem anualmente devido ao trabalho. Isso significa que, todos os dias, quase 7.700 pessoas morrem de doenças ou lesões relacionadas ao trabalho. A cada 15 segundos, no mundo, um trabalhador morre de um acidente ou doença relacionada ao trabalho, e 153 pessoas sofrem algum tipo de lesão. Para Teti et al. 2(018), na Indústria da Construção Civil (ICC) as principais atividades que envolvem os acidentes estudados, são: construções, sendo enfatizadas as verticais; as instalações, que variam as suas atividades, mas se encontram em vários processos da obra; e os reparos, bastante corriqueiros em edificações mais antigas e que possuem necessidade de reforma.

Ainda de acordo com estimativas da mesma instituição, em todo o mundo, o custo total de doenças, ferimentos e mortes foram de $3,94 \%$ do produto interno bruto (PIB) global, ou cerca de 2,99 trilhões de dólares, em custos diretos e indiretos de lesões e doenças, situações que em muitos casos poderiam ser evitados com investimento em Segurança e Saúde do Trabalho (ILO, 2018).

No Brasil, o Anuário Estatístico da Previdência Social - AEPS, publicação que reúne dados sobre diversos aspectos da Previdência Social, incluindo acidentes do trabalho, registrou em 2017 um total de 549.405 acidentes, sendo destes, 30.025 apenas na Indústria da Construção (BRASIL, 2017).

Vasconcelos (2013) coletou e analisou o relatório de 1328 acidentes de trabalho graves e fatais em países como Brasil, Canadá, Estados Unidos, Portugal e Singapura e verificou que um total de $60,8 \%$ de acidentes entre os analisados que poderiam ser evitados através do projeto.

Do total de acidentes analisados, 35,1\% poderiam ser evitados a partir de projetos de concepção (arquitetônico, estruturas e instalações), 27,2\% dos acidentes ser evitados a partir de projetos de execução (como projetos de escoramento, forma, alvenaria e de canteiro como sinalização, acessos, movimentação e armazenamento de materiais, projetos de segurança como andaimes, linhas de vida etc.) e 9,6\% dos acidentes poderiam ser evitados através de projetos de equipamentos (VASCONCELOS, 2013), como gruas, guincho, plataforma elevada motorizada, serras etc.

O Building Information Model - BIM vai muito além da criação de modelos 3D digitais de edifícios com informações incorporadas desde o projeto até a construção e operação. Desta forma o BIM pode ser uma importante ferramenta, auxiliando na tomada de decisões principalmente durante a fase de projeto (KARMARDEEN, 2010). 
Algumas extensões do BIM foram criadas ao longo de sua evolução, de forma a criar várias dimensões (nD), desde a concepção (3D), construção (simulação de planeamento - 4D), estimativa de custos (5D), sustentabilidade e desempenho energético (6D), e gestão de ativos (7D) (ESTRADA, 2015). De acordo com Karmardeen (2010) a segurança pode ser considerada como a oitava dimensão (8D) nos modelos BIM, de forma a apoiar a prevenção através do projeto - prevention through design.

Através da plataforma BIM, é possível ampliar e melhorar a gestão em segurança do trabalho em canteiros de obra da construção Civil, apesar disso a aplicação desta ferramenta continua sendo pouco utilizada para esta finalidade (MANTA et. al, 2018). O objetivo deste artigo é avaliar a percepção e utilização do BIM como ferramenta de gestão em Segurança e Saúde do Trabalho pelos profissionais de Engenharia de Segurança do Trabalho.

\section{MATERIAIS E MÉTODOS}

\subsection{Análise de dados}

O processamento dos dados aconteceu por meio de uma licença estudantil do software SmartPLS 3.0 e do Microsoft Excel 2016.

Para buscar entender o panorama de informação e as relações de importância do Uso do BIM como ferramenta de gestão na SST o presente estudo buscou explicar através da elaboração de um modelo para responder duas hipóteses sobre o tema:

- Hl: Quanto maior a percepção dos benefícios do BIM mais positivas tendem a ser as razões de sua implantação na gestão da SST.

- H2: Quanto maior a percepção das barreiras do BIM mais positivas tendem a ser as razões de sua implantação na gestão da SST.

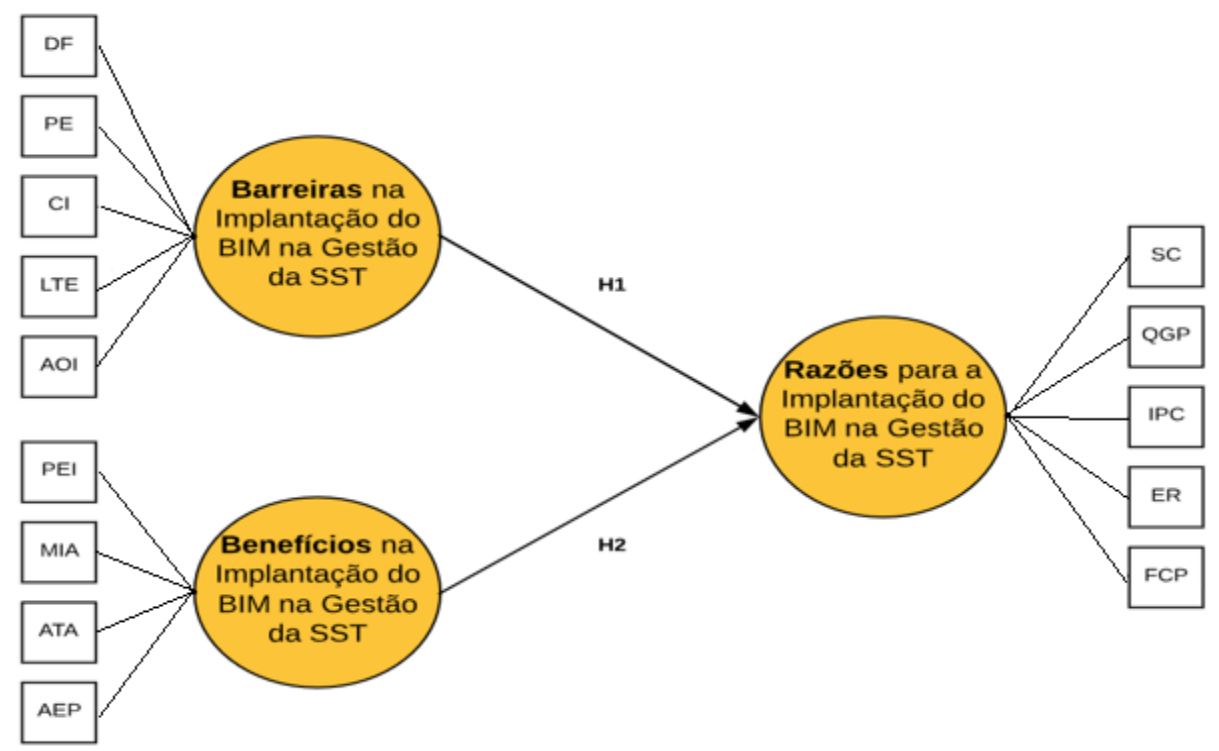

Figura 1 - Modelo de mensuração hipotético -

Fonte: Autores (2019)

Para investigar e tentar provar essas hipóteses um modelo de interação com multivariáveis foi desenvolvido seguindo os critérios do método do Partial Least 
Square (PLS) que combina um número de técnicas estatísticas (CAMPBELL; NTOBEDZI, 2007).

O PLS é uma técnica muito popular para a criação de Modelos de Equações Estruturais (SEM) sendo referida como uma forma de modelagem suave e com uma demanda pequena, ao se considerar as escalas medidas, o tamanho amostral e distribuições residuais (MONECKE; LEISCH, 2012).

As hipóteses e validade do modelo foram testados utilizando uma licença estudantil do software SmartPLS 3.0 e o Microsoft Excel 2016. A Figura 1 e o Quadro 1 mostram de maneira resumida a forma organizacional do modelo proposto.

\section{Quadro 1 - Constructos e variáveis}

\begin{tabular}{|c|c|c|}
\hline Constructos & Variáveis & Descrição \\
\hline \multirow{4}{*}{ Razões } & SC & Satisfação do Cliente \\
\cline { 2 - 3 } & QGP & Qualidade e garantia do produto final \\
\cline { 2 - 3 } & IPC & Interferências positivas no Custo ao Longo Prazo \\
\cline { 2 - 3 } & ER & Eliminação de Riscos \\
\cline { 2 - 3 } & FCP & Facilitar a Compatibilização de Projetos \\
\hline \multirow{5}{*}{ Barreiras } & DF & Desconhecimento da Ferramenta \\
\cline { 2 - 3 } & PE & Política da Empresa \\
\cline { 2 - 3 } & CI & Custo de Implantação \\
\cline { 2 - 3 } & LTE & Limitação Técnica da Equipe \\
\cline { 2 - 3 } & AOI & Ausência de Obrigatoriedade de Implantação \\
\hline \multirow{5}{*}{ Benefícios } & PEI & Possibilidade de Implantação em outros Setores \\
\cline { 2 - 3 } & MIA & Melhoria da Imagem da Empresa \\
\cline { 2 - 3 } & ATA & Auxilio na Tomada de Decisões Estratégicas \\
\cline { 2 - 3 } & AEP & Aumento da Eficiência Produtiva dos Funcionários \\
\hline
\end{tabular}

\subsection{Questionário}

Para determinar os valores numéricos dos indicadores foi elaborado um questionário com perguntas de modo a determinar o nível de conhecimento e utilização do BIM por parte dos profissionais. A segunda parte do questionário avaliou razões, benefícios e barreiras para implementação do BIM através da escala de atitudes Likert (LIKERT, 1932) que mensura o nível de concordância com determinada proposição, para mensurar os indicadores.

\subsection{Participantes}

A amostra consiste em 54 engenheiros de segurança do trabalho formados ou em formação pela Escola Politécnica da Universidade de Pernambuco (POLIUPE). A escolha da POLI como população do estudo se deve ao fato de a instituição possuir reconhecimento na área de segurança ocupacional e ser referência na formação de engenheiros de Segurança do Trabalho no estado de Pernambuco, sendo um dos cursos mais antigos em operação na região. 


\subsection{Coleta de dados}

A coleta dos dados seguiu determinação probabilística, ou seja, a mesma se deu com a determinação da população a ser estudada, que consistiu de 550 indivíduos que passaram ou estão cursando a Especialização em Engenharia de Segurança do Trabalho na POLI entre os anos de 2007 e 2018. De forma a garantir um nível de confiança de $95 \%$ com uma margem de erro de $5 \%$ foi seguido o procedimento de cálculo amostral a seguir:

$$
N=\frac{\delta^{2} \times p \times q}{E^{2}}=\frac{2^{2} \times 50 \times 50}{5^{2}}=400
$$

onde, $\mathrm{N}=$ Tamanho da amostra;

$\delta^{2}=$ nível de confiança de $95 \%$ em número de desvios (Sigmas) indicador de consumo;

$\mathrm{p}=$ proporção do universo com as características pesquisadas da população $(100-p)$;

$\mathrm{q}=$ Proporção do universo que não possui as características pesquisadas.

Com o valor $\mathrm{N}$ definido, foi atribuído um número variando de 1 a 550 para todos os indivíduos e os 400 números foram selecionados aleatoriamente para o envio do questionário via formulário online do Google, por e-mail.

\section{RESULTADOS E DISCUSSÃO}

\subsection{Amostragem}

Um total de 54 indivíduos completaram o questionário da pesquisa no período que a pesquisa ficou disponível. Como o valor obtido ficou muito abaixo dos 400 necessários para garantir os $95 \%$ de confiança com $5 \%$ de margem de erro, um novo cálculo foi feito levando em conta os valores obtidos, chegando-se aos valores da Quadro 2.

Quadro 2 - Parâmetros da Amostragem

\begin{tabular}{|c|c|}
\hline Nível de confiança & Erro amostral \\
\hline $90 \%$ & $10,91 \%$ \\
\hline
\end{tabular}

Fonte: Autores (2019)

\subsection{Resultados qualitativos do questionário}

A primeira parte do questionário, avaliou o conhecimento e nível de envolvimento dos profissionais de Segurança do Trabalho em relação ao BIM. A Figura 2, questiona os participantes se já ouviram falar da ferramenta BIM.

Entre os participantes da pesquisa, a maioria $72,70 \%$ afirmaram já ter ouvido ao menos falar do que é BIM, enquanto os restantes $27,30 \%$ dos profissionais não conhecem a ferramenta. 
Em seguida, foi questionado entre os participantes que já ouviram falar do BIM, caso eles conheciam o BIM 8D. Através do gráfico da Figura 3, pode-se perceber que apenas $24 \%$ tinham conhecimento da existência do BIM 8D.

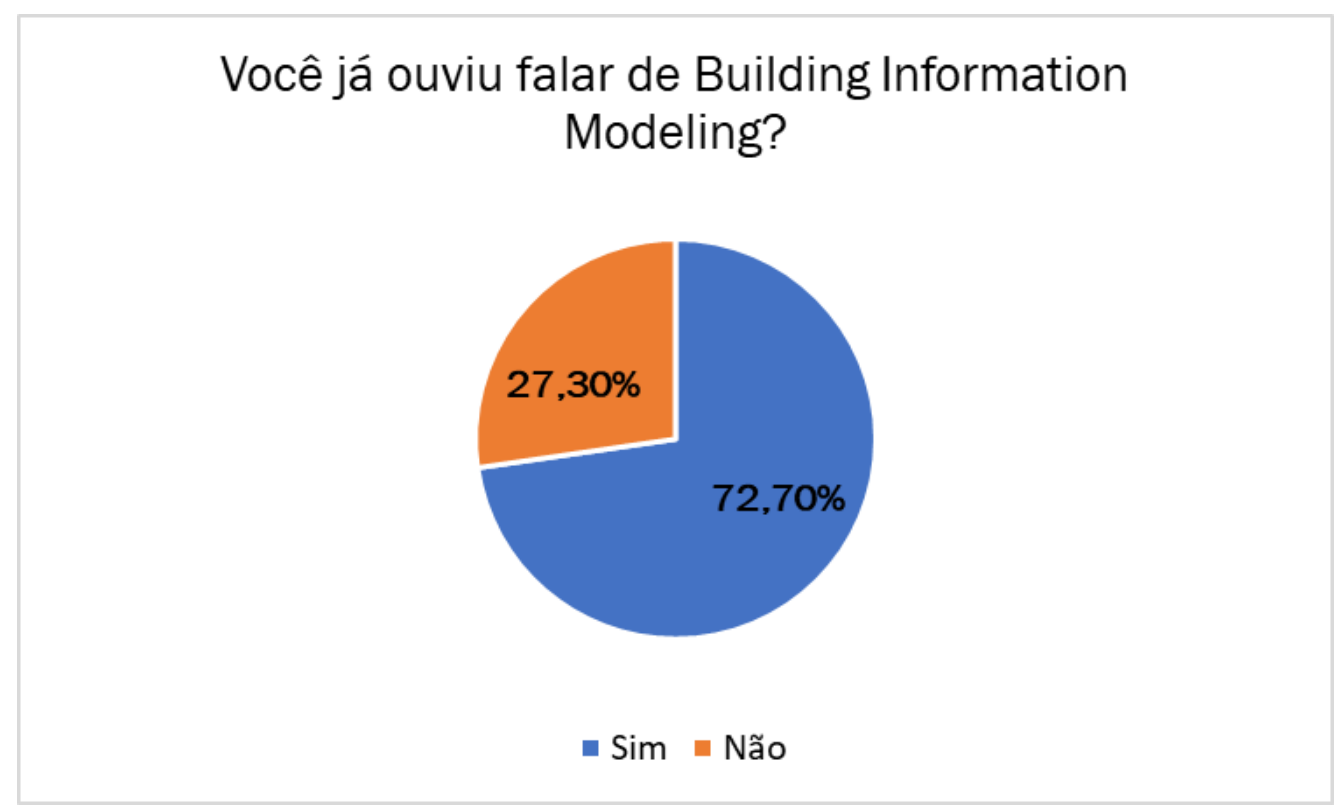

Figura 2 - Questionamento sobre o conhecimento do BIM Fonte: Autores (2019)

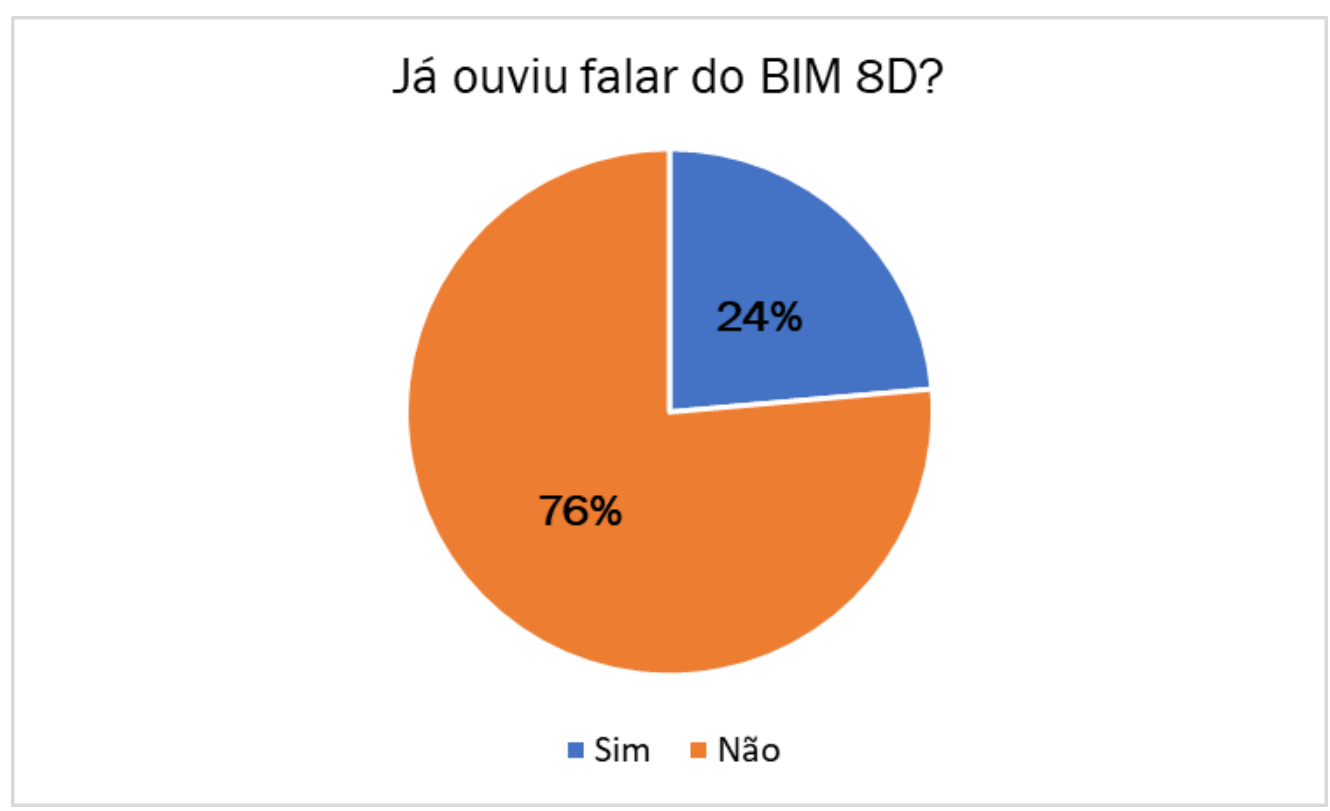

Figura 3 - Questionamento sobre o conhecimento do BIM 8D Fonte: Autores (2019)

Quanto à capacitação e preparação para a utilização do BIM, o gráfico da Figura 4 mostra que $18,20 \%$ dos entrevistados afirmam estar se preparando para a utilização e os avanços da implantação da tecnologia BIM.

O gráfico da Figura 5 mostra que 14,5\% dos entrevistados trabalham ou já trabalharam com o BIM. 
Você está se preparando para o uso do BIM?

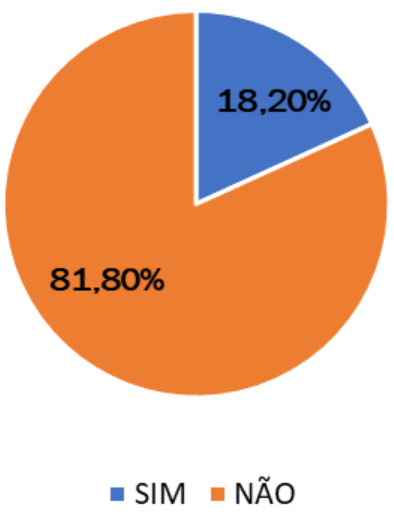

Figura 4 - Questionamento sobre a preparação dos participantes para a ferramenta BIM -

Fonte: Autores (2019)

Você ou sua empresa, trabalham ou já trabalharam com a tecnologia BIM

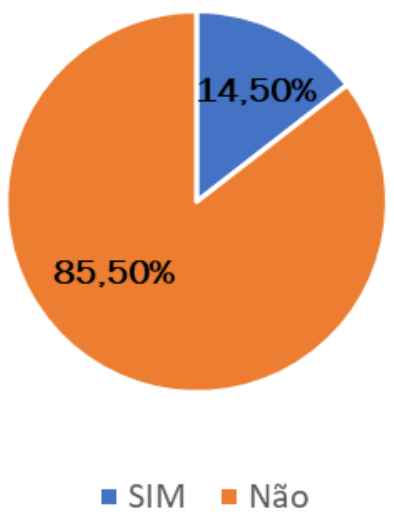

Figura 5 - Questionamento quanto ao uso da tecnologia BIM Fonte: Autores (2019)

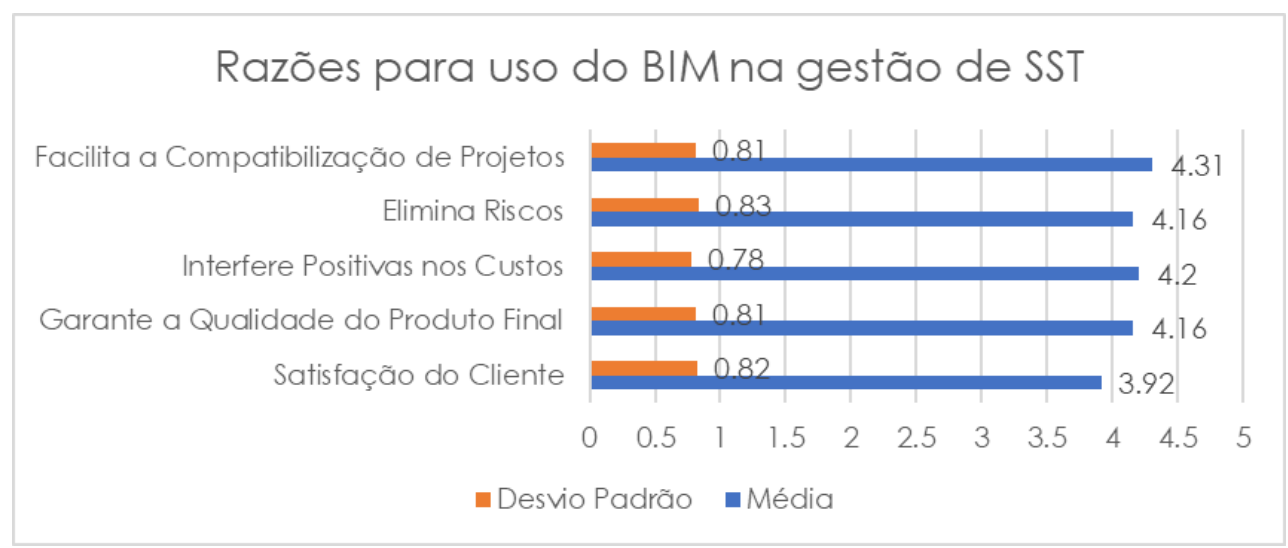

Figura 6 - Razões para implantação do BIM na Gestão da SST Fonte: Autores (2019) 


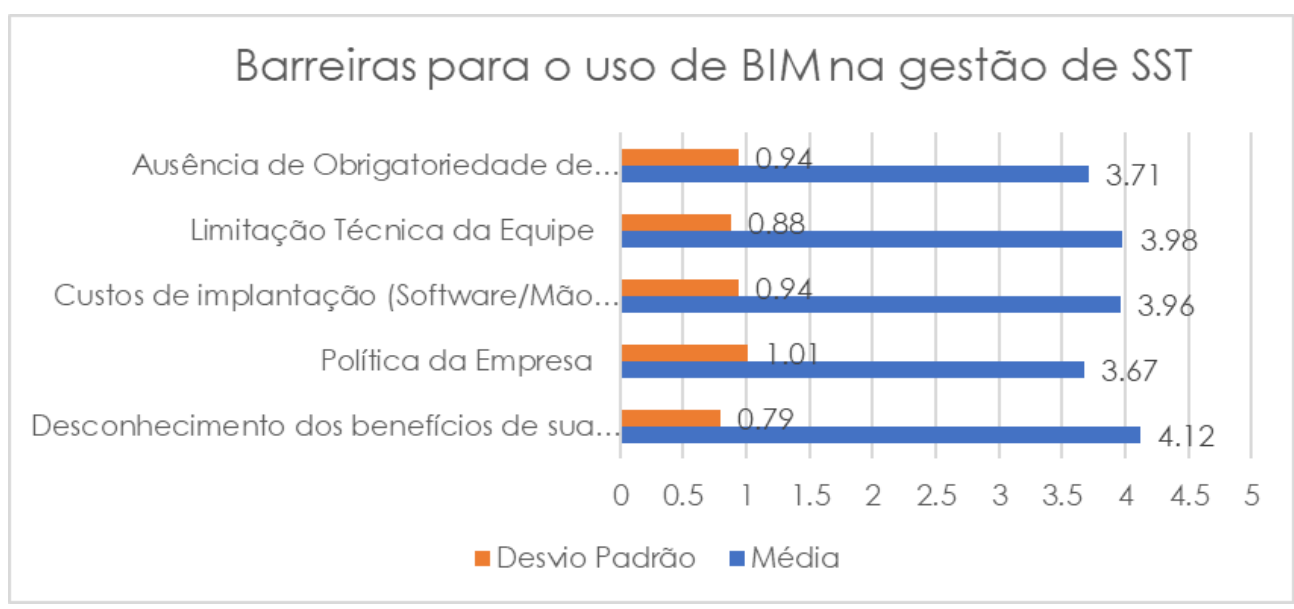

Figura 7 - Barreiras para implantação do BIM na Gestão da SST Fonte: Autores (2019)

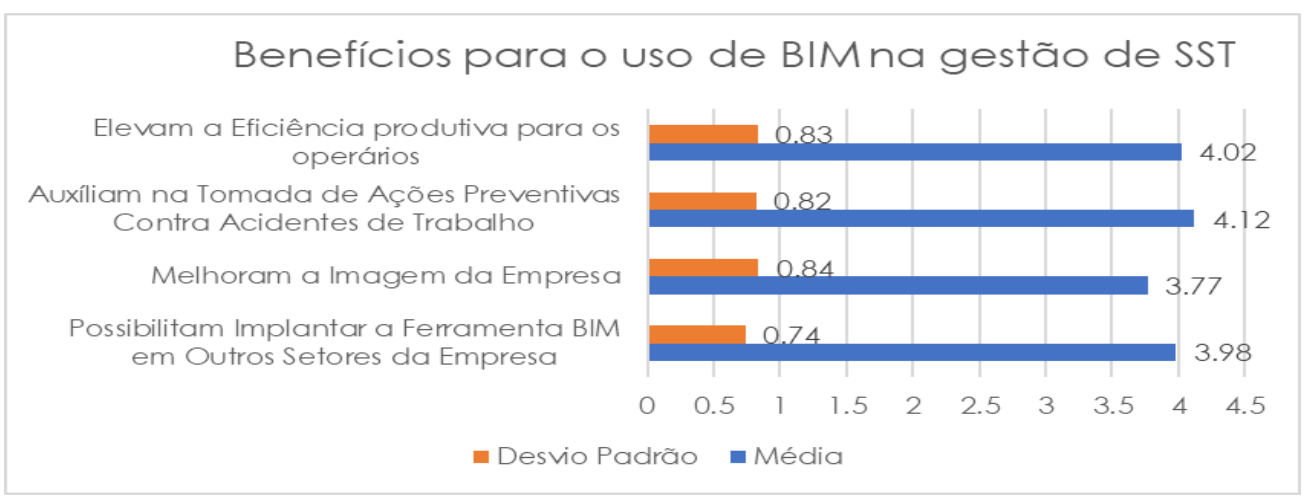

Figura 8 - Benefícios para implantação do BIM na Gestão da SST Fonte: Autores (2019)

As Figuras 6, 7 e 8 mostram os resultados do questionário quantitativo sobre o BIM na Gestão da SST quanto as razões, barreiras e benefícios de se utilizar tão ferramenta. Os resultados demonstram pouca variação nos quesitos relativos as Razões e no que diz respeito aos Benefícios a seção referente as barreiras foi a que mais apresentou oscilações o que pôde ser observado na estruturação do modelo.

\subsection{Avaliação do modelo}

Nesse modelo de mensuração exposto na Figura 1, que se baseia na premissa que os constructos sejam formativos, serão verificadas as validades convergente e discriminante, a confiabilidade dos indicadores e do modelo. $O$ Quadro 3 resume os valores encontrados para o presente modelo.

Com os resultados é possível avaliar os valores:

- Validação convergente: De acordo com o critério de Fornell e Larcker (1981) os valores apresentados para AVE têm que ser superiores a 0,50 ou (50\%) os valores apresentados demonstram que os constructos Benefícios e Razões apresentaram valores acima atestando sua confiabilidade enquanto que o Barreiras apresentou valor inferior;

- Validação Discriminante: De acordo com o critério de Fornell e Larcker (1981) o valor das raízes quadradas dos AVE tem que ser maior que a 
correlação dos mesmos e o mesmo resultado foi encontrado sendo reprovando somente o constructo Barreiras;

- Confiabilidade dos Indicadores: Para que os indicadores apresentem confiabilidade ao modelo os mesmos têm que apresentar valores $>0,7$ (HAIR JR et al., 2016), 4 indicadores do constructo Barreiras ficaram abaixo apresentando falta de confiabilidade para os indicadores.

Quadro 3 - Resultado dos Parâmetros da Amostragem

\begin{tabular}{|c|c|c|c|c|}
\hline Constructos & Variáveis & $\begin{array}{l}\text { Confiabilidade } \\
\text { do Indicador }\end{array}$ & $\begin{array}{l}\text { Confiabilidade } \\
\text { do Constructo }\end{array}$ & $\begin{array}{l}\text { Variância Média Extraída } \\
\text { (AVE) }\end{array}$ \\
\hline \multirow{5}{*}{$\begin{array}{l}\text { Barreiras/Implantação } \\
\text { do BIM na Gestão da } \\
\text { SST }\end{array}$} & DF & 0,552 & \multirow{5}{*}{0,629} & \multirow{5}{*}{0,265} \\
\hline & $\mathrm{PE}$ & 0,566 & & \\
\hline & $\mathrm{Cl}$ & 0,656 & & \\
\hline & LTE & 0,336 & & \\
\hline & $\mathrm{AOI}$ & 0,003 & & \\
\hline \multirow{5}{*}{$\begin{array}{c}\text { Benefícios/Implantação } \\
\text { do BIM na Gestão da } \\
\text { SST }\end{array}$} & PEI & 0,893 & \multirow{5}{*}{0,760} & \multirow{5}{*}{0,552} \\
\hline & MIA & 0,761 & & \\
\hline & ATA & 0,939 & & \\
\hline & AEP & 0,914 & & \\
\hline & SC & 0,825 & & \\
\hline \multirow{4}{*}{$\begin{array}{l}\text { Razões/Implantação } \\
\text { do BIM na Gestão da } \\
\text { SST }\end{array}$} & QGP & 0,885 & \multirow[t]{4}{*}{0,932} & \multirow[t]{4}{*}{0,732} \\
\hline & IPC & 0,835 & & \\
\hline & ER & 0,888 & & \\
\hline & FCP & 0,844 & & \\
\hline
\end{tabular}

Fonte: Autores (2019)

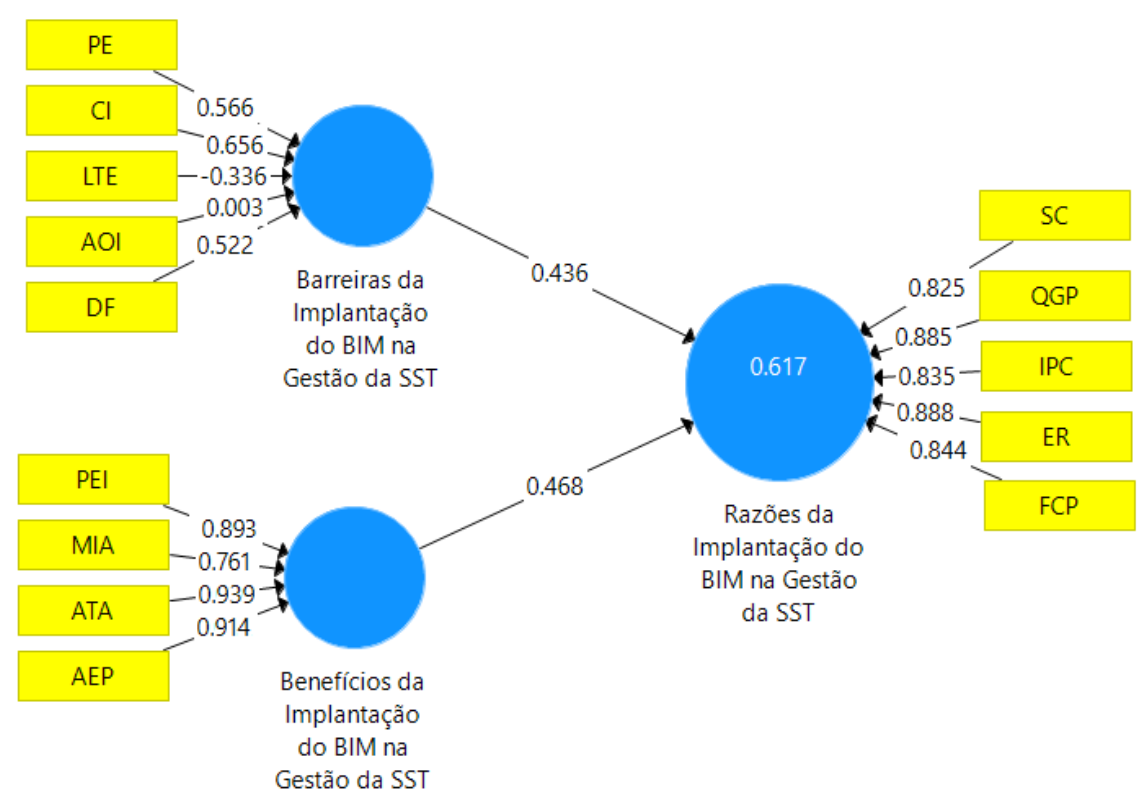

Figura 9 - Resultado do modelo estrutural Fonte: Autores (2019)

Para a confiabilidade do Modelo basicamente três critérios tem que ser avaliados segundo Hair Jr et al. (2016): A determinação do coeficiente de 
determinação ou $\left(R^{2}\right)$; coeficientes do caminho $(\beta)$ e os Valores Estatísticos $T$, que para o nível de significância de $(0,05)$ é 1,96. A partir da Figura 9, gerada no software SmartPLS 3.0, é possível observar a forma de interação do modelo e o valor obtido para o $R^{2}$ que foi de 0,617 ou $61,7 \%$.

Para os coeficientes de caminho ( $\beta$ ) (ou Path Coefficients) contabilizarem impacto considerável para o modelo precisam apresentar valores entre - 1 e +1 (HAIR JR et al., 2016) através do quadro 4 é possível ver os valores para o Valor Estatísticos T e os coeficientes de caminho ( $\beta$ ).

\section{Quadro 4 - Coeficientes de Caminho, Valores Estatísticos T para todos as hipóteses}

\begin{tabular}{|c|c|c|}
\hline Caminho da Hipótese & $\begin{array}{c}\text { Coeficiente de Caminho } \\
(\boldsymbol{\beta})\end{array}$ & Valor Estatístico T \\
\hline Barreiras $\rightarrow$ Razões & 0,456 & 2,035 \\
\hline Benefícios $\rightarrow$ Razões & 0,449 & 1,961 \\
\hline
\end{tabular}

Fonte: Autores (2019)

Desta forma, os resultados dos Valores Estatísticos $\mathrm{T}$, os coeficientes de caminho $(\beta)$ e $R^{2}$ encontrados no quadro 4 atestam para a validade do modelo e das hipóteses.

\section{CONCLUSÕES}

O BIM 8D pode ser uma importante ferramenta de auxílio na gestão em Segurança do trabalho. A partir da pesquisa $72,70 \%$ dos Engenheiros de Segurança entrevistados conhecem O BIM mas, entre estes, apenas $24 \%$ tinham informações sobre o BIM 8D. Além disso, percebe-se que poucos são os profissionais $(18,20 \%)$ que estão investindo e preparando-se para a utilização desta ferramenta e apenas 14,5\% declararam já ter trabalhado com BIM.

O modelo estrutural analisado apresenta validade e as hipóteses apresenta sustentação, porém como a análise não se restringiu somente ao modelo mas também a avaliação dos indicadores de confiança, a afirmação não se confirma em função da hipótese $(\mathrm{H} 2)$, ou seja para os presentes dados a influência da percepção nas Barreiras não influenciam nas Razões da Implantação do BIM na gestão da SST.

Através do modelo é possível perceber que houve influência significativa e positiva das variáveis dos Benefícios sobre as Razões de Implantação do BIM na gestão da SST, sendo que quanto maior/melhores as percepções dos benefícios, maiores tendem a ser as razões da implantação do BIM na gestão da SST. Quanto ao constructo Barreiras pouquíssima ou nenhuma influência de sua percepção, foi comprovada pelo modelo.

O valor de 0,617 encontrado para o coeficiente de regressão $\left(R^{2}\right)$ identifica que as variáveis do constructo Benefícios podem explicar $61,7 \%$ da variabilidade dos resultados do constructo Razões, ou seja, demonstrando o que a interferência dos Benefícios para o incremento das Razões. Ainda assim o valor é considerado estatisticamente fraco, porém é contundente para estudos dessa natureza de acordo com Hair Jr et al. (2016).

A falta de confiança encontrada para os indicadores do construto Barreiras e valor fraco para $\circ \mathrm{R}^{2}(<0,7$ ou $70 \%)$ podem servir como indicativos de que 0 questionário e identificador de variáveis selecionados para esse constructo 
não estejam bem ajustados, sendo necessária melhor refinamento nessa seção, em novos estudos.

\section{REFERÊNCIAS}

BRASIL. MINISTÉRIO DO TRABALHO EPREVIDÊNCIA SOCIAL. Anuário estatístico de acidentes de trabalho 2017. Brasília. 2017. Disponível em:

<http://www.previdencia.gov.br/dados-abertos/dados-abertos-sst/>. Acesso em: 21 out. 2018.

CAMPBELL, A.; NTOBEDZI, A. Emotional intelligence, coping and psychological distress: a partial least squares approach to developing a predictive model. EJournal of Applied Psychology, v. 3, p. 39-54, 2007.

ESTRADA, J. G. Prevenção de riscos na fase de projeto com base na metodologia BIM. 2015. 112 p. Dissertação (Mestrado) - Universidade de Aveiro, Aveiro, 2015.

FORNELL, C.; LARCKER, D. F. Structural equation models with unobservable variables and measurement error: Algebra and statistics. Journal of Marketing Research, v. 18, n. 3, p. 328-388. 1981.

INTERNATIONAL LABOR ORGANIZATION. Safety and health at work. Disponível em: <https://www.ilo.org/global/topics/safety-and-health-at-work/lang-en/index.htm>. Acesso em: out. 2018.

KARMARDEEN, Y. 8D BIM modeling tool for accident prevention through design. IN: EGBU, C. (Ed.). PROCS 26TH ANNUAL ARCOM CONFERENCE, 2010. Anais... Leeds: Association of Researchers in Construction Management, 2010, p. 281 289.

LIKERT, R. A technique for the measurement of attitudes. Archives of psychology. V.22, n, 140. 1932.

MANTA, R.C.; DA CRUZ F.M.; ZLATAR, T.; BARKOKÉBAS JUNIOR, B. THE BIM PLATFORM AND FALLS FROM HEIGHT: THE RISK MANAGEMENT PHASES TO CONSIDER. In: ZLATAR, T.; BARKOKÉKAS JUNIOR, B. (Org.). Building Information Modeling as a Safety Management Tool for Preventing Falls from Height. 1ed., 2018, v. I, p. 48-57.

MONECKE, A.; LEISCH, F. SemPLS: structural equation modeling using partial least squares. Journal of Statistical Software, v. 48, n. 3, p. 1-32, 2012.

HAIR JR, J. F.; HULT, G. T. M.; RINGLE, C.; SARSTEDT, M. A primer on partial least squares structural equation modeling (PLS-SEM). Sage Publications, 2016.

TETI, B.; CRUZ, F. M.; VALERIO, B. M. V.; LAGO, E. M. G.; ZLATAR, T.; BARKOKEBAS JUNIOR, B. WORKING AT HEIGHTS: AN INVESTIGATION ON ACCIDENTS AND ITS CAUSES. In: ZLATAR, T.; BARKOKÉKAS JUNIOR, B. (Org.). Building Information Modeling as a Safety Management Tool for Preventing Falls from Height. led., 2018, v. I, p. 10-.21.

VASCONCELOS, B. M. V. Segurança no Trabalho na Construção: Modelo de gestão de prevenção de acidentes para a fase de concepção. 2013. 181 p. Tese (Doutorado) - Faculdade de Engenharia da Universidade do Porto, Porto, 2013. 